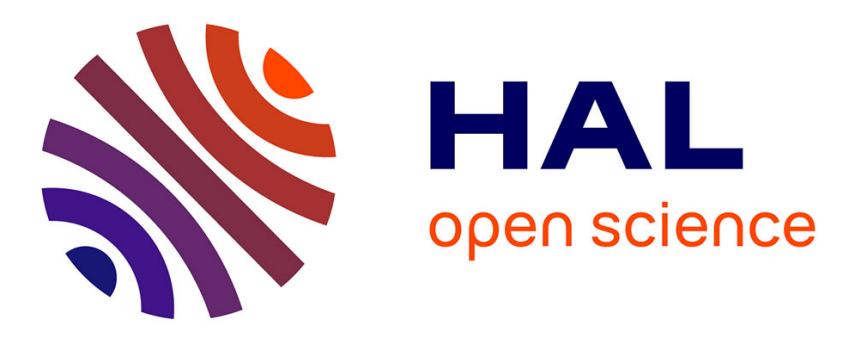

\title{
Study of thermally induced light scattering in a relaxing fluid
}

\author{
J.A. Cowen, C. Allain, P. Lallemand
}

\section{To cite this version:}

J.A. Cowen, C. Allain, P. Lallemand. Study of thermally induced light scattering in a relaxing fluid. Journal de Physique Lettres, 1976, 37 (11), pp.313-316. 10.1051/jphyslet:019760037011031300 . jpa00231300

\section{HAL Id: jpa-00231300 https://hal.science/jpa-00231300}

Submitted on 1 Jan 1976

HAL is a multi-disciplinary open access archive for the deposit and dissemination of scientific research documents, whether they are published or not. The documents may come from teaching and research institutions in France or abroad, or from public or private research centers.
L'archive ouverte pluridisciplinaire HAL, est destinée au dépôt et à la diffusion de documents scientifiques de niveau recherche, publiés ou non, émanant des établissements d'enseignement et de recherche français ou étrangers, des laboratoires publics ou privés. 


\title{
STUDY OF THERMALLY INDUCED LIGHT SCATTERING IN A RELAXING FLUID
}

\author{
J. A. COWEN (*), C. ALLAIN and P. LALLEMAND \\ Laboratoire de Spectroscopie Hertzienne de l'E.N.S., \\ 24 rue Lhomond, 75231 Paris Cedex 05, France
}

(Reçu le 28 juillet 1976, accepté le 15 septembre 1976)

\begin{abstract}
Résumé. - Après un bref rappel sur les équations du mouvement que satisfont les excitations longitudinales dans un fluide qui présente un seul temps de relaxation, on calcule les constantes d'amortissement et les amplitudes des modes Rayleigh et Mountain pour le retour à l'équilibre d'un fluide dans lequel on a induit un réseau thermique par application d'une courte impulsion lumineuse de forte intensité. On présente ensuite des résultats expérimentaux préliminaires obtenus dans la glycérine, qu'on a trouvés être en accord qualitatif avec les prédictions théoriques. On déduit des données expérimentales que la dispersion de la chaleur spécifique est probablement peu importante dans la glycérine.
\end{abstract}

\begin{abstract}
After recalling briefly the equations of motion for longitudinal excitations in a singly relaxing fluid, we calculate the damping constants and amplitudes of the Rayleigh and Mountain modes for the experimental situation in which a thermal grating has been induced in the fluid by a short and intense light pulse. We then present preliminary experimental results obtained in glycerol which agree qualitatively with the theoretical discussion. We derive from the data that the dispersion of the specific heat is probably quite small in glycerol.
\end{abstract}

Various optical techniques are available to study the dynamical behaviour of longitudinal excitations in fluids. Although they all yield the same information concerning the frequency or damping constants of the excitations, the relative contributions of the normal modes of the fluid are quite different in different experiments. We consider here the case of a fluid which exhibits a single structural relaxation time and will calculate the light scattering and forced Rayleigh scattering. We then compare this simple calculation with preliminary experimental results obtained in glycerol in order to demonstrate the main features of forced Rayleigh scattering in a relaxing fluid.

Since light scattering in relaxing fluids has been discussed at lenght by many authors [1-5], we shall recall here only the basic principles of this kind of measurement. Using modern optical techniques, one measures the first order correlation function of the scattered field, which is directly related to the density autocorrelation function

$$
G(\mathbf{K}, t)=\left\langle\rho(\mathbf{K}, t) \rho^{*}(\mathbf{K}, 0)\right\rangle
$$

where $\mathbf{K}$ is the scattering wavevector, whose magnitude is $K=2 K_{\mathrm{L}} n \sin \theta / 2, K_{\mathrm{L}}$ being the wavevector of the incoming light beam, $n$ the index of refraction of the medium and $\theta$ the scattering angle. To calculate $G(K, t)$, we must determine the density at time $t$ knowing what it was at time $t=0$. This can be done using the linearized hydrodynamic equations of the system, which for a singly relaxing fluid (of relaxation time $\tau$ ) can be written, following Mountain [2], as :

$$
\left.\begin{array}{ccc}
\rho_{0} & 0 & 0 \\
b & -\rho_{0} A_{\xi \rho} \frac{K^{2}}{m} & -C_{0}^{2} \beta_{\mathrm{T}} \frac{K^{2}}{\gamma} \\
0 & \frac{1}{\tau} & 0 \\
\frac{\gamma-1}{\beta_{\mathrm{T}}} & 0 & a
\end{array}\right]\left[\begin{array}{l}
\rho \\
\psi \\
\xi \\
T
\end{array}\right]
$$

$\left.{ }^{*}\right)$ On leave from Michigan State University, East Lansing, Michigan, U.S.A. 
This formulation assumes that the slow variables of the system are the density, the velocity gradient along $K$, the temperature and an internal parameter $\xi$. Here

$$
b=\left(\frac{4}{3} \eta_{\mathrm{s}}+\eta_{\mathrm{b}}\right) \frac{K^{2}}{\rho_{0}} \quad \text { and } \quad a=\frac{\lambda K^{2}}{\rho_{0} C_{\mathrm{v}}}
$$

are related to the sound attenuation and to heat diffusion. $\beta_{\mathrm{T}}$ is the thermal expansion coefficient, $\gamma=C_{\mathrm{p}} / C_{\mathrm{v}}$ is the ratio of the specific heats. $C_{0}$ and $C_{\infty}$ are the low and high frequency speeds of sound. $L$ and $A_{\xi \rho}$ are two parameters such that :

$$
\rho_{0} \frac{L A_{\xi \rho}^{2}}{m}=\frac{C_{\infty}^{2}-C_{0}^{2}}{\rho_{0} \tau} .
$$

We remind that Mountain [2] shows that

$$
A_{\xi \rho}=-A_{\xi \xi} \bar{\xi}_{\rho}
$$

using the thermodynamic derivatives :

$$
A_{\xi \xi}=\left(\partial^{2} A / \partial \xi^{2}\right)_{\rho, \mathrm{T}} \text { and } \bar{\xi}=(\partial \xi / \partial \rho)_{\mathrm{T}} .
$$

Note that this formulation assumes that we are studying only the case of pure structural relaxation, but it has been shown previously [6] to be adequate to describe supercooled glycerol which has been used in the experiments discussed below.

To find the correlation function $G(t)$, we take a temporal Laplace transform of this set of equations and express

$$
G(s)=\frac{N(s)}{D(s)}=\sum_{i} A_{i} /\left(s-s_{i}\right),
$$

where $N(s)$ and $D(s)$ are polynomials in $s$. From this we get

$$
G(t)=\sum_{i} A_{i} \mathrm{e}^{s_{i} t} .
$$

In the limit of long relaxation times $\left(C_{0} K \tau \gg 1\right)$, we keep only that part of the dispersion equation $(D(s)=0)$ that leads to the Rayleigh and Mountain modes : one obtains

$$
D(s) \approx s^{2}+s \frac{C_{0}^{2}}{C_{\infty}^{2}}\left[\frac{1}{\tau}+a\left(\frac{1}{\gamma}+\frac{C_{\infty}^{2}-C_{0}^{2}}{C_{0}^{2}}\right)\right]+a \frac{C_{0}^{2}}{C_{\infty}^{2} \gamma \tau} .
$$

The amplitudes of the modes are then easily obtained taking into account the proper initial conditions of the experiments.

For light scattering, we recall that the choice of variables used here is such that $\left\langle\rho(0) \xi^{*}(0)\right\rangle \neq 0$, so that we have to calculate both $\left\langle\rho(t) \rho^{*}(0)\right\rangle$ and $\left\langle\rho(t) \xi^{*}(0)\right\rangle$. This has been discussed previously for the Rayleigh-Mountain coupling range $[5,6]$. It involved a reduced numerator

$$
N(s)=s+\left(a \frac{C_{\infty}^{2}-C_{0}^{2}}{C_{0}^{2}}+\frac{\gamma-1}{\gamma \tau}\right) /\left(\frac{C_{\infty}^{2}}{C_{0}^{2}}-\frac{1}{\gamma}\right) .
$$

Matters are different for the forced Rayleigh technique. In this method, first used by Pohl et al. [7], a spatially modulated light beam is applied to the sample for a short time, in order to produce a temperature grating in the fluid. In turn, this gives rise to a density grating which can be detected by diffraction of a laser beam, as is done in the numerous studies of holographic recording [8] in various materials. To interpret such experiments in a relaxing fluid, we must calculate the correlation function

$$
\left\langle\rho(t) T^{*}(0)\right\rangle .
$$

Assuming that the heating pulse is of such a short duration, so that $\xi(0)=0$, and starting from the same set of equations as above, we find for the numerator (again for $C_{0} K \tau \gg 1$ )

$$
N(s)=s+1 / \tau
$$

If $s_{1}$ and $s_{2}$ are the roots of the reduced dispersion equation, we obtain for the amplitude of the corresponding modes,

$$
\text { and } \quad \begin{aligned}
A_{1} & =\left(s_{1}+1 / \tau\right) /\left(s_{1}-s_{2}\right) \\
A_{2} & =\left(s_{2}+1 / \tau\right) /\left(s_{2}-s_{1}\right) .
\end{aligned}
$$



Fig. 1. - Plot of the variation vs relaxation time of the damping constants (- - - , in log scale at right) and of the amplitude of the Rayleigh and Mountain modes (- in linear scale at left; note that an interchange of labeling occurs for $\tau=1$ ).

Figure 1 shows the variation of $s_{1}, s_{2}, A_{1}$ and $A_{2}$ with respect to $\tau$, with $\gamma=1.15 ; C_{\infty}^{2} / C_{0}^{2}=2$ which are typical values for glycerol. For the limiting case of long or short relaxation times, we find

1) $a \tau \ll 1$ low frequency situation noted « 0 »

$$
\begin{aligned}
s_{\mathrm{R}}=-\frac{a}{\gamma} & s_{\mathrm{M}}=-\frac{C_{0}^{2}}{C_{\infty}^{2} \tau} \\
A_{\mathrm{R}}=\frac{C_{\infty}^{2}}{C_{0}^{2}} & A_{\mathrm{M}}=\left(\frac{C_{0}^{2}}{C_{\infty}^{2}}-1\right) \frac{C_{\infty}^{2}}{C_{0}^{2}} ;
\end{aligned}
$$


2) $a \tau \gg 1$ high frequency situation noted « $\infty »$

$$
\begin{aligned}
& s_{\mathrm{R}}=-\frac{C_{0}^{2}}{C_{\infty}^{2}}\left(\frac{1}{\gamma}+\frac{C_{\infty}^{2}-C_{0}^{2}}{C_{0}^{2}}\right) a \\
& A_{\mathrm{R}}=1 \\
& s_{\mathrm{M}}=-\frac{1}{\gamma \tau\left(\frac{1}{\gamma}+\frac{C_{\infty}^{2}-C_{0}^{2}}{C_{0}^{2}}\right)} \\
& A_{\mathrm{M}}=0 .
\end{aligned}
$$

Note that in the low frequency situation

$$
\left(\tau \ll \rho C_{\mathrm{v}} / \lambda K^{2}\right),
$$

which holds either for small $K$ or small $\tau$ at high temperature, the amplitude of the fastly decaying Mountain line is negative, so that the decay curve will first increase with a time constant of the order of $\tau$ and then decay to zero with a time constant of the order of $\rho C_{\mathrm{v}} / \lambda K^{2}$.

A possible physical interpretation of this feature is to say that as $\tau$ is short, the structure of the fluid has time to come to equilibrium during the duration of the heating pulse. Thus some energy is stored in the structure of the liquid, and it decays into heat within a time $\tau$,' thus yielding an initial increase in the temperature change that is detected by the holographic method discussed in the experimental section of the letter.

There are two interesting features : 1) the ratio $s_{\mathrm{R}}^{\infty} / s_{\mathrm{R}}^{0}$ is equal [6] to $C_{\mathrm{p}}^{0} / C_{\mathrm{p}}^{\infty} ; 2$ ) at low temperature $(a \tau \gg 1)$ the Rayleigh line dominates, in contrast to what was found for the light scattering situation [6]. We shall thus be able to use the forced Rayleigh experiment to measure $s_{R}$ on either side of the coupling region, and thus determine the ratio of the specific heats $C_{\mathrm{p}}^{0} / C_{\mathrm{p}}^{\infty}$.

Note that the related technique of thermal blooming [9], that yields good results in pure fluids [10], gives data that are difficult to interpret in a relaxing fluid due to the non trivial $K$-dependence of the roots of the dispersion equation and of the corresponding amplitudes.

For completeness, let us now consider a third experimental situation, which most probably cannot be used to study structural relaxation, but which is very fruitfully used in the case of thermal relaxation [11], that is to excite the molecular vibrational levels either by infrared absorption or by stimulated Raman scattering. In this case, the correlation function to be considered is $\left\langle\rho(t) \xi^{*}(0)\right\rangle$, and we can show that the corresponding reduced numerator is

$$
N(s)=s+a .
$$

The limiting values of the intensities are now :

$$
\begin{aligned}
a \tau \ll 1 & A_{\mathrm{R}}=0 \quad A_{\mathrm{M}}=1 \\
a \tau \gg 1 & A_{\mathrm{R}}=1-\frac{C_{\infty}^{2}}{C_{0}^{2}\left[\frac{1}{\gamma}+\frac{C_{\infty}^{2}-C_{0}^{2}}{C_{0}^{2}}\right]} \\
A_{\mathrm{M}} & =\frac{C_{\infty}^{2}}{C_{0}^{2}\left[\frac{1}{\gamma}+\frac{C_{\infty}^{2}-C_{0}^{2}}{C_{0}^{2}}\right]} .
\end{aligned}
$$

Experimental. - In order to produce forced Rayleigh scattering, one needs a relatively high powered laser which is absorbed by the relaxing fluid and a relatively low powered one which is not absorbed to probe the index grating. Glycerol was doped with a small amount of iodine to produce a sample which adsorbed approximately $50 \%$ of the $488 \mathrm{~nm}$ line of an argon ion laser in a $5 \mathrm{~mm}$ path. The beam was split into two roughly equal parts which were recombined to interfere in the cryostat containing the glycerol. The angle between the two beams could be chosen so that $K$ varied between 200 and $1000 \mathrm{~cm}^{-1}$. The heating beam was mechanically chopped into $150 \mu$ s pulses, with a $40 \mathrm{~ms}$ repetition rate. The monitor beam from a $2 \mathrm{~mW} \mathrm{He-Ne} \mathrm{laser} \mathrm{is}$ focussed into the sample and is diffracted by the index grating present in the liquid. The diffracted signal is detected by a photomultiplier and averaged over many periods by a multichannel analyzer to improve the signal to noise ratio.

Measurements at room temperature allowed us to verify the $\mathrm{K}^{-2}$ dependence of the Rayleigh mode, confirming earlier results [12], and to optimize the conditions for heterodyne detection. It turns out that there is normally adequate stray light to act as a local oscillator for heterodyne detection, but that it is possible to minimize the stray light so that the initial-large-signal is in the homodyne, but the later-small-signal is in the heterodyne mode. Needless to say, this is not a desirable experimental situation.

There are two novel results of these preliminary experiments. First, we have observed for the first time a negative contribution to $\langle\rho(t) T(0)\rangle$ (initial increase in signal intensity after the end of the heating pulse), due to the Mountain mode. This is shown in figure 2 where at high $\left(-42.5^{\circ} \mathrm{C} ; 2 a\right)$ and low $\left(-70{ }^{\circ} \mathrm{C} ; 2 c\right)$ temperature, only the Rayleigh mode is observed, whereas in the intermediate range $\left(-60.5^{\circ} \mathrm{C} ; 2 b\right)$ a negative contribution is observed. In fact a negative contribution should be detected at high temperature, but it is masked by the finite length $T$ of the heating pulse. The index change varies now as :

$$
\Delta n(t)=\frac{A_{\mathrm{R}}}{s_{\mathrm{R}}}\left(1-\mathrm{e}^{s_{\mathrm{R}} \mathrm{T}}\right) \mathrm{e}^{s_{\mathrm{R}} t}+\frac{A_{\mathrm{M}}}{s_{\mathrm{M}}}\left(1-\mathrm{e}^{s_{\mathrm{M}} \mathrm{T}}\right) \mathrm{e}^{s_{\mathrm{M}} t}
$$



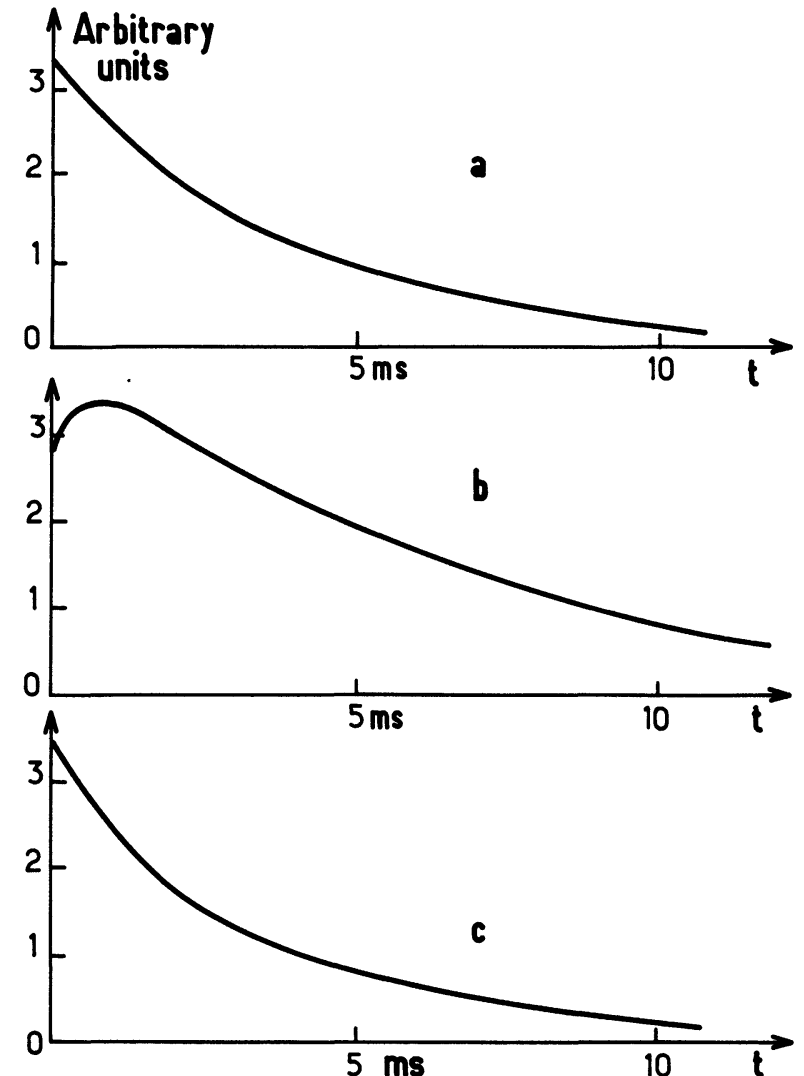

Fig. 2. - Typical signals obtained by the forced Rayleigh technique in glycerol. Here $K=420 \mathrm{~cm}^{-1}$. In $a, T=-42.5^{\circ} \mathrm{C}$; in $b$, $T=60.5^{\circ} \mathrm{C}$; in $c, T=-70^{\circ} \mathrm{C}$.

where $t$ is measured from the end of the heating pulse.

Second, we find that the high and low temperature limits of the mean decay time have approximately the same values. Assuming only slight temperature dependences [13] of $\rho, C_{\mathrm{v}}$ and $\lambda$, this means that $C_{\mathrm{p}}^{0} / C_{\mathrm{p}}^{\infty}$ is close to unity. This confirms in a direct fashion the indirect results that we previously obtained [14]. In addition the temperature dependence of the mean decay time, plotted in figure 3, follows qualitatively the theoretical result obtained for the simplified one relaxation time model discuss-

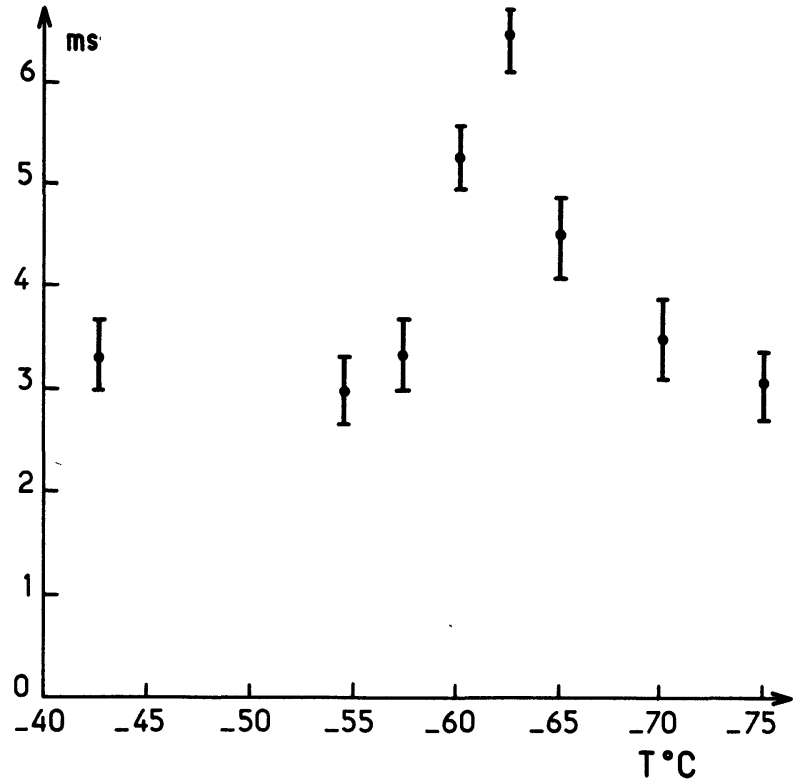

Fig. 3. - Temperature dependence of the mean decay time of the forced Rayleigh scattering signal in glycerol for $K=420 \mathrm{~cm}^{-1}$.

ed above : a meaningful comparison of experiment and theory for the exact value of the increase in mean decay time will be performed when a more complete model is worked out, using the proper distribution of relaxation times for the bulk viscosity of glycerol.

In conclusion, we have shown that by choosing different initial conditions in relaxation studies, the relative contributions of the modes of a fluid can be changed significantly. In particular, we have shown that only the forced Rayleigh scattering technique can be used to study the Rayleigh mode in a relaxing fluid under conditions such that the Rayleigh linewidth is much larger than the Mountain linewidth. This has allowed us to determine that the dispersion of the specific heat of glycerol is quite small, thus supporting our previous conclusions $[6,14]$ concerning the structural nature of the relaxation in glycerol. A more detailed study is in progress to take into account the fact that glycerol exhibits a distribution of relaxation times.

\section{References}

[1] Mountain, R. D., J. Res. Nat. Bur. Stand. 70A (1966) 207.

[2] Mountàin, R. D., J. Res. Nat. Bur. Stand. 72A (1968) 95.

[3] Gornall, W. S., Stegeman, G. I. A., StoichefF, B. P., StoLEN, R. H. and Volterra, V., Phys. Rev. Lett. 17 (1966) 297.

[4] Pinnow, D. A., Candau, S. J., La Macchia, J. T. and Litovitz, T. A., J. Acoust. Soc. Am. 43 (1968) 131.

[5] Allain-Demoulin, C., Lallemand, P. and Ostrowsky, N., Mol. Phys. 31 (1976) 581.

[6] Allain-Demoulin, C., Cazabat, A. M., lallemand, P. and Ostrowsky, N., Opt. Commun. 15 (1975) 126.

[7] Poht, D. W., Schwarz, S. E. and Irniger, V., Phys. Rev. Lett. 31 (1973) 32
[8] Glass, A. M. in "Photonics ", edited by M. Balkanski and P. Lallemand (Gauthier-Villars, Paris) 1975.

[9] Gordon, J. P., Leite, R. C., Moore, R. S., Porto, S. P. and WhinNery, J. R., J. Appl. Phys. 36 (1965) 3.

[10] Calmettes, P. and LaJ, C., J. Physique Colloq. 33 (1972) C1-125.

[11] Ducuing, J., Joffrin, C. and Coffinet, J. P., Opt. Commun. 2 (1970) 6.

AudiberT, M. A., Thèse, Université Paris-Sud, 1976.

[12] Eichler, H., Salje, G. and Stahl, H., J. Appl. Phys. 44 (1973) 5383.

[13] Schultz, A. K., J. Chim. Phys. 51 (1954) 47.

[14] Allain, C. and Cazabat, A. M., Opt. Commun. 16 (1976) 133. 